\title{
ON THE ORDER OF AUTOMORPHISM GROUP OF A COMPACT BORDERED RIEMANN SURFACE OF GENUS FOUR
}

\author{
Dedicated to Professor Mitsuru Ozawa on his 60th birthday
}

\author{
BY TAKAO KATO
}

$\S$ 0. Introduction. For non-negative integers $g$ and $k(2 g+k-1 \geqq 2)$, let $N(g, k)$ be the maximum of the orders of the automorphism groups of compact bordered Riemann surfaces of genus $g$ having $k$ boundary components. Oikawa [9] proved that every automorphism group of a compact bordered Riemann surface is isomorphic to a subgroup of the automorphism group of a compact Riemann surface of the same genus and that $N(g, k)$ is equal to the maximum of the order of the automorphisms groups of $k$-times punctured compact Riemann surfaces of genus $g$. Hurwitz [3] proved that $N(g, 0) \leqq 84(g-1)$. For infinitely many values of $g, N(g, 0)$ were determined by $[1,6,7,8]$. But, for infinitely many $g, N(g, 0)$ are not known. For every $g \geqq 0, N(g, 1), N(g, 2)$ and $N(g, 3)$ were determined by the author [4], for every $k \geqq 0, N(0, k), N(1, k), N(2, k)$ and $N(3, k)$ were determined by $[2,9,11,12]$ and for many other special pairs of $g$ and $k, N(g, k)$ were determined by Ouchi [10]. In this paper we shall determine $N(4, k)$ for every $k \geqq 0$. Wiman [14] showed the equations of all the compact Riemann surfaces of genus 4 which have non-trivial automorphism groups and proved that $N(4,0)=120$. To determine $N(4, k)$, we shall study subgroups of groups which Wiman showed.

The author wishes to represent his thanks to Professor Accola who showed Wiman's paper $[13,14]$ and gave some advice to him and to Mr. Nakagawa who read the manuscript of this paper and pointed out many errors.

§1. Lemmas: Let $S$ be a compact Riemann surface of genus 4 and let $G$ be a group of automorphisms of $S . S / G$ has the conformal structure induced by the conformal structure of $S$ such that the natural projection $\pi$ of $S$ onto $S / G$ is holomorphic. Then, there are at most finite number of points $P_{1}, \cdots, P_{t}$ on $S / G$ over which $\pi$ is ramified with multiplicities $\nu_{1}, \cdots, \nu_{t}\left(\nu_{j} \geqq 2\right)$, respectively. Then Riemann-Hurwitz's relation shows

$$
6 / N=2 \tilde{g}-2+\sum_{j=1}^{t}\left(1-1 / \nu_{j}\right)
$$

Received April 12, 1983 
where $N$ is the order of $G$ and $\tilde{g}$ is the genus of $S / G$. Note that if $\tilde{g}=0$, then $t \geqq 3$ and that $\pi^{-1}(P)$ consists of $N / \nu_{j}$ points if $P=P_{j}(j=1, \cdots, t)$ and $N$ points otherwise. We call such a group $G$ a $\left(\tilde{g} ; \nu_{1}, \cdots, \nu_{t}\right)$ group. For simplicity's sake we shall denote $\left(0 ; \nu_{1}, \cdots, \nu_{t}\right)$ by $\left(\nu_{1}, \cdots, \nu_{t}\right)$.

Using these notations we have a sequence of Lemmas.

LEMma 1. For any point $P$ on $S / G$, all the points of $\pi^{-1}(P)$ have the same Weierstrass gap sequences.

Proof. For any two points $Q_{1}, Q_{2}$ of $\pi^{-1}(P)$, there is an element of $G$, i.e., an automorphism of $S$, which maps $Q_{1}$ to $Q_{2}$.

Lemma 2. Assume $\tilde{g}=0$. Let

$$
k=m N+\sum_{j=1}^{t} \varepsilon_{j}\left(N / \nu_{j}\right),
$$

where $m$ is a non-negative integer and $\varepsilon_{j}=0$ or $1(j=1, \cdots, t)$. Then, $N(4, k) \geqq N$.

Proof. Choose $m$ points $P_{t+1}, \cdots, P_{t+m}$ on $S / G-\left\{P_{1}, \cdots, P_{t}\right\}$ arbitrarily. Delete the set of points $\pi^{-1}\left(P_{j}\right),(j=t+1, \cdots, t+m)$ and the set of points $\pi^{-1}\left(P_{j}\right)$, if $\varepsilon_{j}=1(j=1, \cdots, t)$ from $S$. Then we have a $k$-times punctured Riemann surface of genus 4 such that $G$ is a group of automorphisms of it. Thus $N(4, k) \geqq N$.

LEMma 3. (Hurwitz [3]). Assume i) $\tilde{g} \geqq 1$ and $G$ is not $a(1 ; 2)$ group, or ii) $t \geqq 6$. Then, $N<12$, especially $N \leqq 5$ provided if $N$ is prime.

LEMMA 4. $N$ cannot be divided by any prime number greater than 5 .

Proof. Assume $N$ is divisible by a prime number $N^{\prime} \geqq 7$. Using Sylow's theorem we may assume $N=N^{\prime}$. By Lemma 3 , we have $\tilde{g}=0$. By Lemma 1 , $\nu_{1}=\cdots=\nu_{t}=N$ and $t \geqq 3$. Using Riemann-Hurwitz's relation, we have

$$
6 / N=-2+t(1-1 / N) \text {. }
$$

Hence, $N=(t+6) /(t-2)$ which is a contradiction.

For any point $Q$ of $\pi^{-1}\left(P_{\jmath}\right)(j=1, \cdots, t)$, there is an element $\phi$ of $G$ which fixes $Q$ and generate a cyclic group $\langle\phi\rangle$ of order $\nu_{\jmath}$. Thus we have:

Lemma 5. There is a cyclic subgroup of $G$ of order $\nu_{j}(j=1, \cdots, t)$.

But the order of a cyclic group is restricted from above such as:

Lemma 6. (Wiman [13], Kato [4]). If $G$ is a cyclic group, then $N \leqq 18$.

The next lemma is a well known property of hyperelliptic surface. 
LEMma 7. Assume $S$ is the hyperelliptic surface defined by

$$
y^{2}=\left(x-\alpha_{1}\right)\left(x-\alpha_{2}\right) \cdots\left(x-\alpha_{10}\right) .
$$

Then, every automorphism of $S$ induces a linear transformation of the $x$-sphere which maps the set $\left\{\alpha_{1}, \cdots, \alpha_{10}\right\}$ onto itself. Hence, $N \leqq 40$.

In the following two Lemmas we shall show properties of cyclic trigonal surface of genus 4 .

Lemma 8. (Kato [5]). Assume $S$ is defined by

$$
y^{3}=\left(x-\alpha_{1}\right)\left(x-\alpha_{2}\right) \cdots\left(x-\alpha_{6}\right) .
$$

Then, every automorphism of $S$ induces a linear transformation of the $x$-sphere which maps the set $\left\{\alpha_{1}, \alpha_{2}, \cdots, \alpha_{6}\right\}$ onto itself. Hence, $N \leqq 72$.

Lemma 9. (Kato [5]). Assume $S$ is defined by

$$
y^{3}=\left(x-\alpha_{1}\right)\left(x-\alpha_{2}\right)\left(x-\alpha_{3}\right)\left(x-\alpha_{4}\right)^{2}\left(x-\alpha_{5}\right)^{2}\left(x-\alpha_{6}\right)^{2} .
$$

Then, the number of Weierstrass points whose gap sequences are $\{1,2,4,5\}$ is 6,9 , or 12 . If there are 12 such Weierstrass points, then $N$ is a multiple of 36 , i.e., $N=36$ or 72 .

$\S 2$. Models. In this section we shall list up Riemann surfaces which are used to determine $N(4, k)$ and show some properties of those surfaces.

Let $S_{j}(j=1, \cdots, 19)$ be the Riemann surfaces of genus 4 defined by the equations as follows, throughout these equations $\alpha, \beta$ and $\gamma$ are mutualiy distinct complex numbers which are neither 0 nor 1 :

$$
\begin{aligned}
& S_{1}: y^{5}=x^{3}(x-1)^{2}(x+1), \\
& S_{2}: y^{3}=x\left(x^{4}-1\right), \\
& S_{3}: y^{3}=\left(x^{3}-1\right) /\left(x^{3}+1\right), \\
& S_{4}: y^{2}=x^{10}-1, \\
& S_{5}: y^{2}=x\left(x^{8}-1\right), \\
& S_{6}: y^{2}=x^{9}-1, \\
& S_{7}: y^{3}=x^{6}-1, \\
& S_{8}: y^{5}=x^{3}-1, \\
& S_{9}: y^{6}=x\left(x^{2}-1\right), \\
& S_{10}: y^{4}=x^{3}(x-1)(x-\alpha)(x-\beta),
\end{aligned}
$$




$$
\begin{aligned}
& S_{11}: y^{4}=x^{3}(x-1)(x-\alpha)^{2}(x-\beta)^{2}(x-\gamma)^{2}, \\
& S_{12}: y^{2}=x\left(x^{4}-1\right)\left(x^{4}-\alpha\right), \\
& S_{13}: y^{10}=x(x-1)^{3}, \\
& S_{14}: y^{6}=x(x-1)(x-\alpha)^{2}, \\
& S_{15}: y^{6}=x(x-1)(x-\alpha), \\
& S_{16}: y^{6}=x(x-1)^{2}(x-\alpha)^{4}, \\
& S_{17}: y^{5}=x(x-1)(x-\alpha), \\
& S_{18}: y^{5}=x^{4}(x-1)(x-\alpha), \\
& S_{19}: y^{5}=x^{3}(x-1)^{2}(x-\alpha) .
\end{aligned}
$$

We shall show the automorphism group Aut $S_{\jmath}$ of $S_{\jmath}(j=1, \cdots, 9)$ in the following Properties 1-9.

Property 1. Choose as basis of holomorphic differentials on $S_{1}$ such as $\theta_{1}=d x / y, \quad \theta_{2}=x d x / y^{2}, \quad \theta_{3}=x(x-1) d x / y^{3}$ and $\theta_{4}=x^{2}(x-1) d x / y^{4}$. Then we have a canonical embedding of $S_{1}$ into $\boldsymbol{P}^{3}(\theta)$ with projective coordinates $\left(\theta_{1}, \theta_{2}, \theta_{3}, \theta_{4}\right)$. Embed $\boldsymbol{P}^{3}(\theta)$ into $\boldsymbol{P}^{4}(X)$ such as

$$
\left(\begin{array}{l}
X_{1} \\
X_{2} \\
X_{3} \\
X_{4} \\
X_{5}
\end{array}\right)=\left(\begin{array}{cccc}
1 & 1 & 1 & -1 \\
\eta^{4} & \eta^{3} & \eta^{2} & -\eta \\
\eta^{3} & \eta & \eta^{4} & -\eta^{2} \\
\eta^{2} & \eta^{4} & \eta & -\eta^{3} \\
\eta & \eta^{2} & \eta^{3} & -\eta^{4}
\end{array}\right)\left(\begin{array}{c}
\theta_{1} \\
\theta_{2} \\
\theta_{3} \\
\theta_{4}
\end{array}\right),
$$

where $\eta=e^{2 \pi i / 5}$. Then $\boldsymbol{P}^{3}(\theta)$ is mapped onto the hyperplane $X_{1}+X_{2}+X_{3}+X_{4}+X_{5}$ $=0$ in $\boldsymbol{P}^{4}(X)$ and the image of $S_{1}$ is mapped onto the intersection of the hyperplane and two hypersurfaces,

$$
\begin{aligned}
& X_{1}^{2}+X_{2}^{2}+X_{3}^{2}+X_{4}^{2}+X_{5}^{2}=0, \\
& X_{1}^{3}+X_{2}^{3}+X_{3}^{3}+X_{4}^{3}+X_{5}^{3}=0 .
\end{aligned}
$$

This is known as Bring's curve [14] and its automorphism group is of order 120 which is a $(2,4,5)$ group. Let $\phi_{\jmath}(j=1,2)$ be the automorphisms of $\boldsymbol{P}^{4}(X)$ defined by

$$
\begin{aligned}
& \phi_{1}:\left(X_{1}, X_{2}, X_{3}, X_{4}, X_{5}\right) \longrightarrow\left(X_{2}, X_{1}, X_{3}, X_{4}, X_{5}\right), \\
& \phi_{2}:\left(X_{1}, X_{2}, X_{3}, X_{4}, X_{5}\right) \longrightarrow\left(X_{1}, X_{3}, X_{4}, X_{5}, X_{2}\right) .
\end{aligned}
$$

Let $\phi_{1 j}(j=1,2)$ be the automorphisms of $S_{1}$ corresponding to $\dot{\varphi}_{j}$. Then, Aut $S_{1}=\left\langle\phi_{11}, \phi_{12}\right\rangle$ and $\phi_{13}=\phi_{11}^{\circ} \phi_{12}$ is of order 5 . 
Property 2. Let $\phi_{21}, \phi_{22}$ and $\phi_{23}$ be the automorphisms of $S_{2}$ defined by

$$
\begin{aligned}
& \phi_{21}:(x, y) \longrightarrow\left(e^{\pi i / 2} / x, e^{\pi i / 2} y / x^{2}\right), \\
& \phi_{22}:(x, y) \longrightarrow\left(\frac{i-x}{i+x}, 2 e^{\pi \imath / 6} y /(x+i)^{2}\right), \\
& \phi_{23}=\phi_{22}{ }^{\circ} \phi_{21} .
\end{aligned}
$$

Then the orders of $\phi_{21}, \phi_{22}$ and $\phi_{23}$ are $2,3,12$, respectively and $\left\langle\phi_{21}, \phi_{22}\right\rangle$ is a $(2,3,12)$ group of order 72 . By Lemma 8 we have Aut $S_{2}=\left\langle\phi_{21}, \phi_{22}\right\rangle$.

Property 3. Let $\phi_{31}, \phi_{32}$ and $\phi_{33}$ be the automorphisms of $S_{3}$ defined by

$$
\begin{aligned}
& \phi_{31}:(x, y) \longrightarrow(1 / x,-y), \\
& \phi_{32}:(x, y) \longrightarrow\left(y,-e^{2 \pi i / 3} / x\right), \\
& \phi_{33}=\phi_{32}{ }^{\circ} \phi_{31} .
\end{aligned}
$$

Then, the order of $\phi_{31}, \phi_{32}$ and $\phi_{33}$ are 2, 4 and 6, respectively, and $\left\langle\phi_{31}, \phi_{32}\right\rangle$ is a $(2,4,6)$ group of order 72 . Since $N(4,0)=120$, Aut $S_{3}=\left\langle\phi_{31}, \phi_{32}\right\rangle$.

Property 4. Let $\phi_{41}, \phi_{42}$ and $\phi_{43}$ be the automorphisms of $S_{4}$ defined by

$$
\begin{aligned}
& \phi_{41}:(x, y) \longrightarrow\left(e^{\pi i / 5} / x, e^{\pi i / 2} y / x^{5}\right), \\
& \phi_{42}:(x, y) \longrightarrow\left(1 / x, e^{\pi i / 2} y / x^{5}\right), \\
& \phi_{43}=\phi_{41} \circ \phi_{42} .
\end{aligned}
$$

Then, the orders of $\phi_{41}, \phi_{42}$ and $\phi_{43}$ are 2, 4 and 10, respectively. Since $S_{4}$ is hyperelliptic, by Lemma 6 we have Aut $S_{4}=\left\langle\phi_{41}, \phi_{42}\right\rangle$ which is a $(2,4,10)$ group of order 40 .

Property 5. Let $\phi_{51}, \phi_{52}$ and $\phi_{53}$ be the automorphisms of $S_{5}$ defined by

$$
\begin{aligned}
& \phi_{51}:(x, y) \longrightarrow\left(e^{\pi i / 4} / x, e^{5 \pi i / 8} y / x^{5}\right), \\
& \phi_{52}:(x, y) \longrightarrow\left(1 / x, e^{\pi i / 2} y / x^{5}\right), \\
& \phi_{53}=\phi_{51} \circ \phi_{52} .
\end{aligned}
$$

Then the orders of $\phi_{51}, \phi_{52}, \phi_{53}$ are 2,4 and 16, respectively and by Lemma 6 Aut $S_{5}=\left\langle\phi_{51}, \phi_{52}\right\rangle$ which is a $(2,4,16)$ group of order 32 .

Property 6. Let $\phi_{61}$ be the automorphism of $S_{6}$ defined by

$$
\phi_{61}:(x, y) \longrightarrow\left(e^{2 \pi i / 9} x,-y\right) .
$$

Then, by Lemma 6 we have Aut $S_{6}=\left\langle\phi_{61}\right\rangle$ which is a $(2,9,18)$ group of order 18 . 
Property 7. Let $\phi_{71}, \phi_{72}$ and $\phi_{73}$ be the automorphisms of $S_{7}$ defined by

$$
\begin{aligned}
& \phi_{71}:(x, y) \longrightarrow\left(e^{\pi i / 3} / x, e^{\pi i / 3} y / x^{2}\right), \\
& \phi_{72}:(x, y) \longrightarrow\left(e^{\pi i / 3} x, y\right), \\
& \phi_{73}=\phi_{71}{ }^{\circ} \phi_{72} .
\end{aligned}
$$

Then, the orders of $\phi_{71}, \phi_{72}$ and $\phi_{73}$ are 2, 6, 6, respectively, and $\left\langle\phi_{71}, \phi_{72}\right\rangle$ is a $(2,6,6)$ group of order 36. Assume the order of Aut $S_{7}$ is 72 . By Lemma 7, the cubic group is a subgroup of Aut $S_{7}$. But in this case $\left\langle\phi_{71}, \phi_{72}\right\rangle$ induces a dihedral group of the $x$-sphere. It is a contradiction. Hence, Aut $S_{7}=\left\langle\phi_{71}, \phi_{72}\right\rangle$.

Property 8. Let $\phi_{81}$ be the automorphism of $S_{8}$ defined by

$$
\phi_{81}:(x, y) \longrightarrow\left(e^{2 \pi \imath / 3} x, e^{2 \pi \imath / 5} y\right) .
$$

By Lemma 7 we have Aut $S_{8}=\left\langle\phi_{81}\right\rangle$ which is a $(3,5,15)$ group of order 15 .

Property 9. Let $\phi_{91}$ be the automorphism of $S_{9}$ defined by

$$
\phi_{91}:(x, y) \longrightarrow\left(-x, e^{\pi \imath / 6} y\right) .
$$

We have Aut $S_{9}=\left\langle\phi_{91}\right\rangle$ which is a $(4,6,12)$ group of order 12 . In fact, points over $x=0,1,-1$ are Weierstrass points whose gap sequences are $\{1,2,3,7\}$. Hence, meromorphic functions of order 3 on $S_{9}$ are linear fractions in $y$. As a covering of the $y$-sphere, $S_{9}$ has 12 branch points over $y^{12}=4 / 27$. Hence, automorphisms are possibly induced from $y \rightarrow 1 / y, y \rightarrow e^{\pi i / 6} y$ and these compositions. However, the gap sequences of the three points over $y=0$ are $\{1,2,3,7\}$ and those over $y=\infty$ are $\{1,2,3,5\}$. Hence, an automorphism induced from $y \rightarrow 1 / y$ does not exist.

$\S 3$. Estimate of $N(4, k)$. To determine $N(4, k)$, we have to consider the possibility of $\left(\tilde{g}, \nu_{1}, \cdots, \nu_{t}\right)$ group. However, giving an estimate of $N(4, k)$ from below, we shall not need to consider groups of small order.

Proposition (10). $\quad N(4, k) \geqq 10$ for all $k$.

Proof. The group $\left\langle\phi_{72}^{3}, \phi_{73}\right\rangle$ is a $(2,2,3,6)$ group of order 12 . Since every even number can be represented as $12 m+6 \varepsilon_{1}+6 \varepsilon_{2}+4 \varepsilon_{3}+2 \varepsilon_{4}$ by a suitable non-negative integer $m$ and $\varepsilon_{j}=0$ or $1(j=1, \cdots, 4)$, by Lemma 2 we have $N(4, k) \geqq 12$ if $k \equiv 0(\bmod 2) .\left\langle\phi_{42}{ }^{\circ} \phi_{41}\right\rangle$ is a $(5,10,10)$ group of order 10 . Hence, by Lemma 2 , if $k \equiv 0,1,2,3,4(\bmod 10)$, then $N(4, k) \geqq 10 .\left\langle\phi_{42}^{2}, \phi_{43}^{2}\right\rangle$ is a $(2,2,5,5)$ group of order 10 . Hence, again by Lemma 2 , if $k \equiv 0,2,4(\bmod 5)$, then $N(4, k) \geqq 10$. Therefore, we have $N(4, k) \geqq 10$ for all $k$.

Thus, it is not necessary to consider groups of order less than or equal to 10. We shall list up possible groups of order more than 10 . 
Making the following table we are assuming Lemmas 3, 4 and 6.

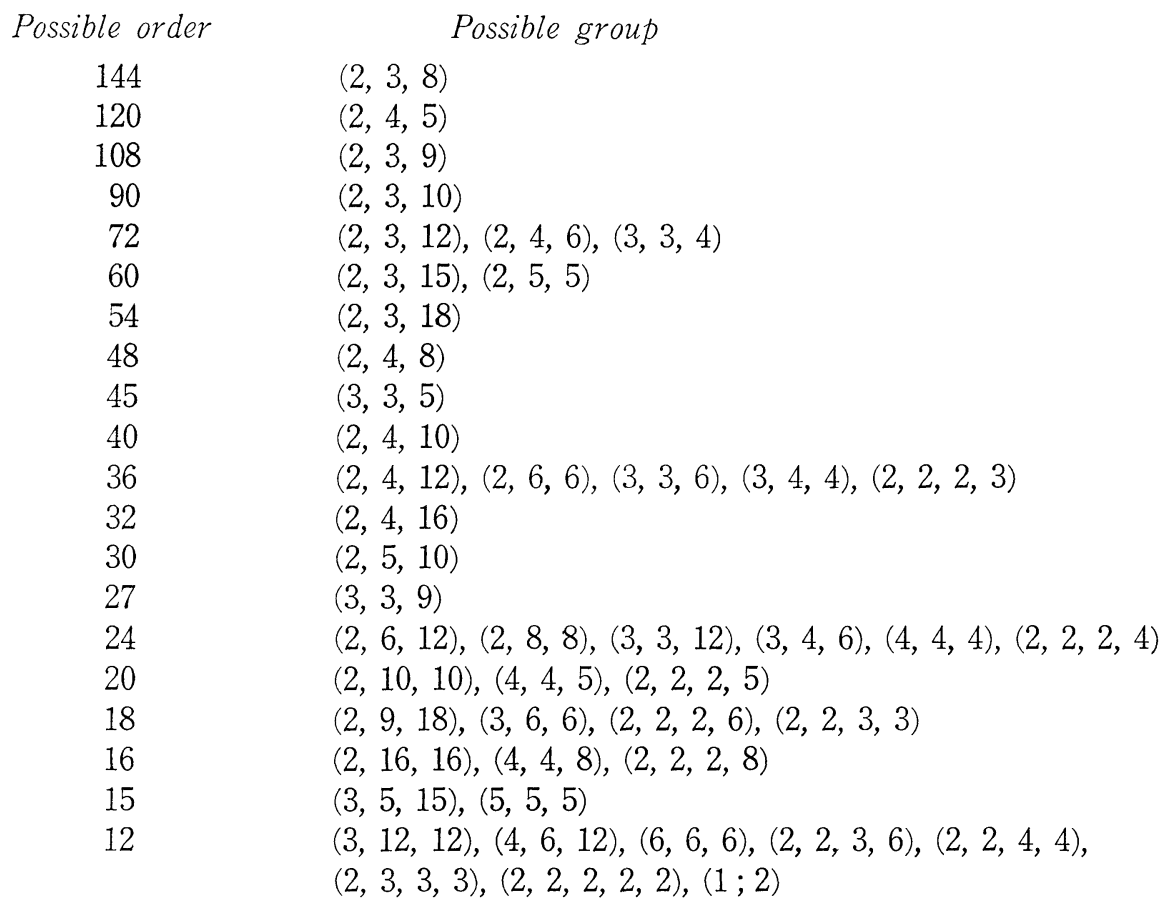

It is known that $N(4,0) \leqq 120$ [4]. Hence, $N(4, k) \leqq 120$ for all $k$. It is also known that an automorphism group of order 108 or 90 does not exist [14]. We shall give an alternative proof of this facts.

Proposition (120). A $(2,3, r)$ group does not exist for $r=8,9$ or 10 . Hence, if $k \equiv 0,24(\bmod 30)$, then $N(4, k)=120$.

Proof. Assume $r=8$. Since the total weights of Weierstrass points on $S$ is 60 , by Lemma 1 , we have

$$
144 \alpha_{1}+72 \alpha_{2}+48 \alpha_{3}+18 \alpha_{4}=60
$$

for some nonnegative integers $\alpha_{1}, \alpha_{2}, \alpha_{3}$ and $\alpha_{4}$. But it is impossible.

Assume $r=9$. By Lemmas 3 and $5, S$ is defined by

$$
y^{9}=x^{\lambda}(x-1)^{\mu}, \quad 3 \nmid \lambda, \mu, \lambda+\mu .
$$

Hence, it is conformally equivalent to $S_{6}$ which is hyperelliptic. Hence, the order of Aut $S$ is 18.

Assume $r=10$. As is the case of $r=8$, there are 30 Weierstrass points of weight 2 . On the other hand, by Lemmas 3 and $5, S$ is conformally equivalent 
to $S_{4}$ or $S_{13}$. But $S_{4}$ is hyperelliptic and $S_{13}$ has a Weierstrass point of weight 4 at $(x, y)=(0,0)$. Contradiction.

Using Lemma 1 and Property 1 , we can prove that if $k \equiv 0,24(\bmod 30)$, then $N(4, k)=120$.

Proposition $(72)$. If $k \equiv 0(\bmod 6)$ and $N(4, k) \neq 120$, then $N(4, k)=72$.

Proof. Using Lemma 2 and Properties 1, 2 and 3, we can prove this Proposition. As a fact, there is no $(3,3,4)$ group. It is proved by Wiman [14]. But we shall give an alternative proof. Assume there is a $(3,3,4)$ group and $\nu_{1}=\nu_{2}=3, \nu_{3}=4$. Then, by Lemma 1 and the fact that the total weights of Weierstrass points is 60 , all the points of $\pi^{-1}\left(P_{3}\right)$ are Weierstrass points of weight 2 and all the points of one of the sets $\pi^{-1}\left(P_{1}\right)$ and $\pi^{-1}\left(P_{2}\right)$, say $\pi^{-1}\left(P_{1}\right)$, are Weierstrass points of weight 1 . The possibilities of gap sequences of Weierstrass points of weight 2 are $\{1,2,4,5\}$ and $\{1,2,3,6\}$. Since there are 18 points in $\pi^{-1}\left(P_{3}\right)$, the gap sequences of these points are $\{1,2,3,6\}$, (cf. Kato [5, Theorem 1]). Assume $\alpha$ is an automorphism of $S$ which fixes a point $Q$ of $\pi^{-1}\left(P_{3}\right)$ and $f$ is a meromorphic function on $S$ which has a pole of order 4 at $Q$ and is holomorphic elsewhere. Then, $\left(f+f \circ \alpha+f \circ \alpha^{2}+f \circ \alpha^{3}\right)^{1 / 4}$ is a single valued meromorphic function on $S /\langle\alpha\rangle$, whose order is 1 . Hence, $S /\langle\alpha\rangle$ is the sphere. Therefore, $S$ is conformally equivalent to $S_{10}$ or $S_{11}$. But $S_{10}$ has a Weierstrass point of weight 3 at $(x, y)=(0,0)$ and $S_{11}$ has a Weierstrass point of weight 6 at $(x, y)=(0,0)$. Both of them contradict our assumption.

Proposition (60). $A(2,3,15)$ group does not exist. For each $k, N(4, k) \neq 60$.

Proof. Assume a subgroup of Aut $S$ is a $(2,3,15)$ group. By Lemma 5, Aut $S$ has an element of order 15. Hence, $S$ is conformally equivalent to $S_{8}$. But the order of Aut $S_{8}$ is 15 . This is a contradiction. Assume $k$ is an integer such that $N(4, k)=60$. Then, by Lemma 2

$$
k=60 m+\varepsilon_{1}(60 / 2)+\varepsilon_{2}(60 / 5)+\varepsilon_{3}(60 / 5),
$$

for a nonnegative integer $m$ and $\varepsilon_{j}=0$ or $1(j=1,2,3)$. Thus, $k \equiv 0(\bmod 6)$. But for such a $k, N(4, k) \geqq 72$ by Proposition (72).

Proposition (54). A $(2,3,18)$ group does not exist.

Proof. Assume a subgroup of Aut $S$ is a $(2,3,18)$ group. Then, there is an automorphism of $S$ of order 18. Hence, $S$ is conformally equivalent to $S_{6}$. But the order of Aut $S_{6}$ is 18 . This is a contradiction.

Proposition (48). A $(2,4,8)$ group does not exist.

Proof. Assume a subgroup of Aut $S$ is a $(2,4,8)$ group. Then, there is an automorphism of $S$ of order 8 and $S$ is conformally equivalent to $S_{12}$. Since $S_{12}$ 
is hyperelliptic, by Lemma 7 we have a contradiction.

Proposition (45). A $(3,3,5)$ group does not exist.

Proof. Assume a subgroup of Aut $S$ is a $(3,3,5)$ group. Then, there is an automorphism of $S$ of order 5 and $S$ is conformally equivalent to $S_{17}, S_{18}$ or $S_{19}$. Assume $S_{17}$ admits a $(3,3,5)$ group $G$ and $P$ is the point of $S_{17} / G$ which corresponds to a fixed point of an automorphism of order 5 . Then, $\pi^{-1}(P)$ consists of 9 points and the points corresponding to $x=0,1, \alpha$ and $\infty$ are in $\pi^{-1}(P)$. However, the gap sequences of these points are $\{1,2,3,7\}$ for $x=0,1, \alpha$ and $\{1,2,4,7\}$ for $x=\infty$. This is a contradiction. Since $S_{18}$ is hyperelliptic, the order of Aut $S_{18}$ is 40 . Assume $S_{19}$ admits a $(3,3,5)$ group. There is an automorphism of $S_{19}$ whose order is 2 . Hence, the order of Aut $S_{19}$ is a multiple of 90. This contradicts Proposition (120).

Proposition $(40)$. If $k \equiv 0,4(\bmod 10)$ and $N(4, k) \neq 120,72$, then $N(4, k)=40$.

Proof. Observe Property 4 and Lemma 2.

Proposition (36). If $k \equiv 9$ or $21(\bmod 36)$, then $N(4, k)=36 . \quad A(2,4,12)$ group does not exist.

Proof. $\left\langle\phi_{33}^{2}, \phi_{32}\right\rangle$ is a $(3,4,4)$ group. Therefore, if $k \equiv 0,9,12,21$, or 30 $(\bmod 36)$, then $N(4, k) \geqq 36$. But if $k \equiv 0,12,18,30(\bmod 36)$, then $N(4, k) \geqq 72$. By virtue of Proposition (72) it is not necessary to consider the possibility of cases $(2,6,6),(3,3,6)$ and $(2,2,2,3)$ groups.

Assume $S$ admits a $(2,4,12)$ group. Then, there is an automorphism of $S$ of order 12 . Hence, $S$ is defined by either

or

$$
y^{12}=x(x-1)^{4}
$$

$$
y^{12}=x(x-1)^{2} .
$$

The former is conformally equivalent to $S_{2}$ and the latter is to $S_{9}$. On $S_{2}$ there are exactly 6 Weierstrass points whose gap sequences are $\{1,2,4,7\}$. Let $Q_{1}, \cdots, Q_{6}$ be these points. Then,

$$
{ }^{\#} \pi^{-1}\left(\left\{\pi\left(Q_{1}\right), \cdots, \pi\left(Q_{6}\right)\right\}\right)=6,
$$

by Lemma 1 . However, it is impossible because 6 cannot be represent as $36 m+(36 / 2) \varepsilon_{1}+(36 / 4) \varepsilon_{2}+(36 / 12) \varepsilon_{3}$ for a nonnegative integer $m$ and $\varepsilon_{j}=0$ or 1 $(j=1,2,3)$. Hence, $S_{2}$ does not admit a $(2,4,12)$ group. On $S_{9}$ there are 3 Weierstrass points whose gap sequences are $\{1,2,4,7\}$. Hence they should be fixed points of automorphisms of order 12. For nonnegative integers $m_{1}, m_{2}$ and $m_{3}, 36 m_{1}+(36 / 2) m_{2}+(36 / 4) m_{3}$ is divisible by 9 . On the other hand the total weight of Weierstrass points except for the above 3 points is 48 which cannot be divided by 9 . Hence, by Lemma $1 S_{9}$ does not admit a $(2,4,12)$ group. 

$=32$.

Proposition (32). If $k \equiv 0,2(\bmod 8)$ and $N(4, k) \neq 120,72,40$, then $N(4, k)$

Proof. Observe Property 5 and Lemma 2.

Proposition (30). A $(2,5,10)$ group does not exist.

Proof. Assume $S$ admits a $(2,5,10)$ group. Then, there is an automorphism of $S$ of order 10. Hence, $S$ is conformally equivalent to $S_{1}$ or $S_{13}$. On $S_{4}$ there are exactly 10 hyperelliptic Weierstrass points. By Lemma $1, S_{4}$ does not admit a $(2,5,10)$ group. On $S_{13}$ the gap sequence corresponding to $x=0$ is $\{1,2,4,7\}$ and that to $x=1$ is $\{1,2,3,6\}$. Both of these points are fixed points of an automorphism of order 10 . Hence, by Lemma $1 S_{13}$ does not admit a $(2,5,10)$ group.

Proposition (27). A $(3,3,9)$ group does not exist.

Proof. Assume $S$ admits a $(3,3,9)$ group. Then $S$ has an automorphism of order 9 . Hence, $S$ is conformally equivalent to $S_{6}$. But the order of Aut $S_{6}$ is 18 . This is a contradiction.

Proposition (24). If $k \equiv 2,4(\bmod 12)$ and $N(4, k) \neq 40,32$, then $N(4, k)=24$. None of the following groups exists: $(2,8,8),(3,3,12)$ and $(3,4,6)$ groups. It is not necessary to consider $(4,4,4)$ and $(2,2,2,4)$ groups.

Proof. $\left\langle\phi_{21}, \phi_{23}^{2}\right\rangle$ is a $(2,6,12)$ group. Hence, by Lemma 2 , if $k \equiv 0,2,4$, or 6 , then $N(4, k) \geqq 24$. By Lemma 2 and Proposition (72), it is not necessary to consider $(4,4,4)$ and $(2,2,2,4)$ groups. Assume $S$ admits a $(2,8,8)$ group. Then $S$ has an automorphism of order 8 and $S$ is conformally equivalent to $S_{12}$. Since $S_{12}$ is hyperelliptic, by Lemma 1 we have a contradiction. Assume $S$ admits a $(3,3,12)$ group. Since $S$ has an automorphism of order $12, S$ is conformally equivalent to $S_{2}$ or $S_{9}$. Observing Weierstrass points whose gap sequences are $\{1,2,4,7\}$, by a similar argument as Proposition (36), we have a contradiction. Assume $S$ admits a $(3,4,6)$ group. Then, $S$ is conformally equivalent to $S_{14}, S_{15}$ or $S_{16}$. $S_{14}$ is conformally equivalent to the surface defined by

$$
y^{3}=x\left(x^{2}-1\right)\left(x^{2}-\alpha /(\alpha-1)\right) .
$$

Hence, $S_{14}$ has 6 Weierstrass points whose gap sequences are $\{1,2,4,7\}$. At least 2 of those points are fixed points of an automorphism of order 6 . Therefore, by Lemma $1 S$ is not conformally equivalent to $S_{14}$. Assume $S$ is conformally equivalent to $S_{15}$. Let $\phi$ be an automorphism of $S_{15}$ defined by

$$
\phi:(x, y) \longrightarrow\left(x, e^{\pi i / 3} y\right) .
$$

Then, $\phi$ has exactly 3 fixed points. On the other hand, there exist 4 points on 
$S_{15}$ which are fixed by automorphisms of order 6 . This is a contradiction. Assume $S$ is conformally equivalent to $S_{16}$. $S_{16}$ is conformally equivalent to the surface defined by

$$
y^{3}=x\left(x^{2}-1\right)\left(x^{2}-\alpha\right)^{2} .
$$

Hence, by Lemma 9 there exist at least 6 Weierstrass points whose gap sequences are $\{1,2,4,5\}$. Especially the fixed points of automorphisms of order 6 are among those points. There are exactly 4 such points. Hence, by Lemma 1 and Lemma 9 the 8 fixed points of automorphisms of order 3 also have the gap sequence $\{1,2,4,5\}$. Therefore, again by Lemma 9, the order of Aut $S_{16}$ is 36 or 72. Since the order of $(3,4,6)$ group is 24 , the order of Aut $S_{16}$ is 72 . Hence, $S$ is conformally equivalent to $S_{3}$. Observing a $(2,4,6)$ group, i.e., Aut $S_{3}$, there, are 12 Weierstrass points of weight 2 which are the fixed points of order 6 and there are either 18 Weierstrass points of weight 2 which are the fixed points of order 4 or 36 Weierstrass points of weight 1 which are the fixed points of order 2 (as a fact, the latter case does occur). But both the cases contradict Lemma 1.

Proposition $(20)$. If $k \equiv 2,5,9,12(\bmod 20)$ and $N(4, k) \neq 72,36,32,24$, then $N(4, k)=20$.

Proof. $\left\langle\phi_{42}^{2}, \phi_{43}\right\rangle$ is a $(2,10,10)$ group and $\left\langle\phi_{42}, \phi_{43}^{2}\right\rangle$ is a $(1,4,5)$ group. Hence, by Lemma 2, we have this proposition.

Proposition $(18)$. If $k \equiv 0(\bmod 3)$ or $k \equiv 1,2(\bmod 9)$ and $N(4, k)=120,72$, $40,36,32,24,20$, then $N(4, k)=18$. It is not necessary to consider $(2,2,2,6)$ groups.

Proof. $\left\langle\phi_{61}\right\rangle$ is a $(2,9,18)$ group, $\left\langle\phi_{73}^{2}, \phi_{72}\right\rangle$ is a $(3,3,6)$ group and $\left\langle\phi_{73}^{3}, \phi_{72}^{2}{ }^{\circ} \phi_{73}^{3},\left(\phi_{72}{ }^{\circ} \phi_{71}\right)^{2}\right\rangle$ is a $(2,2,3,3)$ group. Apply Lemma 2 .

Proposition (16). If $k \equiv 1,4,6(\bmod 16)$ and $N(4, k) \neq 120,72,40,36,24$, 20,18 , then $N(4, k)=16$. It is not necessary to consider $(2,2,2,8)$ groups.

Proof. $\left\langle\phi_{53}\right\rangle$ is a $(2,16,16)$ group and $\left\langle\phi_{52}, \phi_{53}^{2}\right\rangle$ is a $(4,4,8)$ group. Apply Lemma 2.

Proposition (15). If $k \equiv 1,4,5,8(\bmod 15)$ and $N(4, k) \neq 40,32,24,20,18,16$, then $N(4, k)=15$. It is not necessary to consider $(5,5,5)$ groups.

Proof. $\left\langle\phi_{81}\right\rangle$ is a $(3,5,15)$ group. Apply Lemma 2.

Proposition $(12)$. If $k \equiv 1,3,5(\bmod 12)$ or $k \equiv 0(\bmod 2)$ and $N(4, k) \neq 120$, $72,40,32,24,20,18,16,15$, then $N(4, k)=12$. It is not necessary to consider $(6,6,6),(2,2,4,4),(2,3,3,3)$ and $(2,2,2,2,2)$ groups. 
Proof. $\left\langle\phi_{23}\right\rangle$ is a $(3,12,12)$ group and $\left\langle\phi_{91}\right\rangle$ is a $(4,6,12)$ group. Apply Lemma 2.

Summing up these Propositions we have:

THEOREM :

$$
\begin{aligned}
N(4, k)=120, & \text { if } \quad k \equiv 0,24(\bmod 30), \\
72, & \text { if } k \equiv 0(\bmod 6) \text { and } N(4, k) \neq 120, \\
40, & \text { if } k \equiv 0,4(\bmod 10) \text { and } N(4, k) \neq 120,72, \\
36, & \text { if } k \equiv 9,21(\bmod 36), \\
32, & \text { if } k \equiv 0,2(\bmod 8) \text { and } N(4, k) \neq 120,72,40, \\
24, & \text { if } k \equiv 2,4(\bmod 12) \text { and } N(4, k) \neq 40,32, \\
20, & \text { if } k \equiv 2,5,9,12(\bmod 20) \text { and } N(4, k) \neq 72,36,32,24, \\
18, & \text { if } \quad k \equiv 0(\bmod 3) \text { or } k \equiv 1,2(\bmod 9) \text { and } N(4, k) \neq 120, \\
& 72,40,36,32,24,20, \\
16, & \text { if } \quad k \equiv 1,4,6(\bmod 16) \text { and } N(4, k) \neq 120,72,40,36,24, \\
& 20,18, \\
15, & \text { if } \quad k \equiv 1,4,5,8(\bmod 15) \text { and } N(4, k) \neq 40,32,24,20, \\
& 18,16, \\
12, & \text { if } \quad k \equiv 1,3,5(\bmod 12) \text { or } k \equiv 0(\bmod 2) \text { and } N(4, k) \\
& \neq 120,72,40,32,24,20,18,16,15
\end{aligned}
$$

\section{REFERENCES}

[1] AccolA, R.D.M., On the number of automorphisins of a closed Riemann surface, Trans. Amer. Math. Soc. 131 (1968), 398-408.

[2] Heins, M., On the number of 1-1 directly conformal maps which a muitiply-connected plane region of finite connectivity $p(>2)$ admits onto itself, Bull. Amer. Math. Soc. 52 (1946), 454-457.

[3] Hurwi'tz, A., Über algebraische Gebilde mit eindeutigen Transformationen in sich, Math. Ann. 41 (1893), 403-422.

[4] Kato, T., On the number of automorphisms of a compact bordered Riemann surface, Kōdai Math. Sem. Rep. 24 (1972), 224-233.

[5] Kato, T., On Weierstrass points whose first non-gaps are three, Journ. reine angew. Math. 316 (1980), 99-109.

[6] Kiley, W. T., Automorphism groups on compact Riemann surfaces, Trans. Amer. Math. Soc. 150 (1970), 557-563.

[7] Macbeath, A. M., On a theorem of Hurwitz, Proc. Glasgow Math. Assoc. 5 (1961), 90-96.

[8] Maclachlan, C., A bound for the number of automorphisms of a compact Riemann surface, J. London Math. Soc. 44 (1969), 265-272.

[9] Orkawa, K., Note on conformal mappings of a Riemann surface onto itself, Kōdai Math. Sem. Rep. 8 (1956), 23-30, 115-116.

[10] Ouchi, S., The number of conformal automorphisms of a Riemann surface and normal subgroups of modular groups, Master thesis, Tokyo University, (1979). 
(Japanese)

[11] TsujI, R., On conformal mapping of a hyperelliptic Riemann surface onto itself, Kōdai Math. Sem. Rep. 10 (1958), 127-136.

[12] TsUjI, R., Conformal automorphisms of a compact bordered Riemann surface of genus 3, Kōdai Math. Sem. Rep. 27 (1967), 271-290.

[13] Wiman, A., Über die hyperelliptischen Curven und diejenigen vom Geschlechte $p=3$ welche eindeutigen Transformationen in sich zulassen, Bihang Till Kongl. Svenska Veienskaps-Akademiens Hadlingar 21 (1895-96), 1-23.

[14] Wiman, A., Über die algebraischen Curven von den Geschlechtern $p=4,5$ und 6 welche eindeutige transformationen in sich besitzen, Bihang Till Kongl. Svenska Veienskaps-Akademiens Hadlinger 21 (1895-96), afd 1, no. 3, 41 pp.

Department of Mathematics, Brown University and

Department of Mathematics, Yamaguchi University 